\title{
Comparison of the effects of two different dose algorithms in cardiac dose parameters for hodgkin lymphoma patients receiving mediastinal radiotheraphy
}

\author{
Emre Tahberer ${ }^{1}$, Mehmet Adigul ${ }^{2}$, Ahmet Ergin Capar ${ }^{3}$, Ahmet Cinkaya ${ }^{4 *}$, Yasin Coban ${ }^{5}$, \\ Serdar Surenkok ${ }^{1}$
}

\section{Abstract}

Objective: This study aimed to compare pencil beam (PB) and convolution superposition (CS) dose calculation algorithms with respect to cardiac dose-volume parameters V5, V25, V30 and MHD (median heart dose) in Hodgkin lymphoma (HL) patients who received mediastinal radiotherapy (RT).

Material and Methods: Ten eligible cases with the diagnosis of HL who completed mediastinal RT at Dokuz Eylül University Department of Radiation Oncology before 01.01.2011 formed the study population. Related 3D conformal RT treatment plans were obtained from the Treatment Planning System (TPS) (Nucletron Oncentra Master Plan Version 3.3 SP3 program). Calculations were done for 6 and $18 \mathrm{MV}-\mathrm{X}$ beam energy, respectively by using PB and CS algorithms in the TPS. Two algorithms were compared in terms of cardiac dose-volume parameters (V5, V25, V30 and MHD).

Results: Mean relative difference for both V25 and V30 was found to be significantly higher in favor of PB algorithm in both $6 \mathrm{MV}-\mathrm{X}$ and $18 \mathrm{MV}-\mathrm{X}$ photon energy. Mean relative differences were $1.75 \%(\mathrm{p}=0.012)$ and $6.76 \%(p=0.028)$ for the V25 and V30 parameters, respectively in $6 \mathrm{MV}-\mathrm{X}$ and $3.25 \%(\mathrm{p}=0.021)$ and $13.95 \%$ $(\mathrm{p}=0.007)$ for the V25 and V30 parameters, respectively in $18 \mathrm{MV}-\mathrm{X}$ energy.

Conclusion: When PB algorithm is used in RT treatment planning of HL patients for the mediastinal region where tissue inhomogeneity is relatively high, heart V25 and V30 parameters were shown to result in higher values compared to CS algorithm in our study. It is more appropriate to prefer CS algorithm instead of PB algorithm since it increases accuracy of calculations in the treatment planning of mediastinal RT.

Key words: Convolution / superposition, pencil beam, Hodgkin lymphoma, cardiac dose-volume parameters

\section{Introduction}

Radiotherapy (RT), which plays an important role in the traetment of Hodgkin's lymphoma (HL), has complications related with technique, dose and irradiated volume (1).

In HL, bilateral hilar lymhatic regions should always be included in mediastinal RT volume according to the recommendations related to "Involved Field Radiotherapy" (IFRT). Therefore, bilateral lung tissue constitute inhomogeneity together with trachea, main bronchi and mediastinal soft tissue in mediastinal RT. Heart is exposed to a significant portion of the targeted dose in various extent since it is located partially within the RT field.
Thus, mediastinal RT volume in patients with HL is considered to be an appropriate region for the comparison of pencil beam (PB) and convolution/superposition (CS) algorithms. Despite the development of quicker and more sensitive novel diagnostic techniques, their complexity and high cost have limited their use in many poor-resource countries.

Due to the rapidly growing TB problem in these countries, there is an urgent need to assess promising alternative methodologies in settings with high disease prevalence (6).

Received 15-06-2016 Accepted 20-06-2016 Available Online 15-08-2016

1 Acrbadem University, Faculty of Medicine, Department of Radiation Oncology, Istanbul, TR

2 Dokuz Eylül University, Faculty of Medicine, Department of Radiation Oncology, Izmir, TR

3 Tepecik Research and Training Hospital Department of Radiology, Izmir, TR

4 Dumlupınar University, Faculty of Medicine, Department of Radiation Oncology, Kutahya, TR

5 Gaziosmanpaşa University, Faculty of Medicine, Department of Radiation Oncology, Tokat, TR

* Corresponding Author: Ahmet Cinkaya E-mail:drahmetcinkaya@gmail.com Phone: +00905327730720 
In this study, we aimed to examine the relationships between EZN, culture and PCR and to investigate whether an algorithm has been used in the administration of these methods.

\section{Material and Methods}

Ten eligible cases with the diagnosis of HL who completed mediastinal RT at Dokuz Eylül University Department of Radiation Oncology before 01.01.2011 formed the study population. Related 3D conformal RT treatment plans were obtained from the Treatment Planning System (TPS) (Nucletron Oncentra Master Plan Version 3.3 SP3 program). Selection criteria were; being diagnosed as HL, at least $1 / 3$ of the heart being included in the mediastinal RT field (mantle, modified mantle, only mediastinum + bilateral hilar regions), 3dimensional conformal treatment plan being performed in Nucletron Oncentra computed TPS, being treated to a dose of at least $30 \mathrm{~Gy}$. PTV and OAR (Organ at Risk) delineation were performed according to our institution's protocols on the Oncentra MasterPlan image registration module, with CT slice intervals of $5 \mathrm{~mm}$ acquired under normal respiration conditions in the CT-simulator.

Calculations were redone for 6 and $18 \mathrm{MV}-\mathrm{X}$ beam energy, respectively by using PB and CS algorithms in the TPS. For each patient's treatment plan, all plan elements (beam angles, beam energies, beam weights, etc.) were the same in both calculations using two different algorithms. The same reference isodose was chosen for each treatment plan formed with the same beam energy using $\mathrm{PB}$ and CS algorithm. Under these conditions, V5, V25, V30 and median heart dose (MHD) ) values were obtained from DVHs.

Two algorithms were compared in terms of cardiac dose-volume parameters (V5, V25, V30 and median heart dose (MHD) ). Statistical analysis was performed using SPSS 15.0 program. Since the number of patients was below 30, a non-parametric test had to be used. Also, the Wilcoxon signed rank test was preferred due to the comparison of two related data. For statistical significance, the $\mathrm{p}$ value was accepted to be smaller than 0.05 .

\section{Results}

V5, V25, V30 and MHD parameters were documented at the end of calculations done for different algorithms on the plans of the cases. The results related to dose-volume parameters of cases are given for $6 \mathrm{MV}-\mathrm{X}$ and $18 \mathrm{MV}-\mathrm{X}$ in Table 1 and Table 2, respectively.According to the data given in Table 1 and 2, mean V25, V30 and MHD values are higher in PB algorithm, while mean V5 parameter is higher in CS algorithm for both $6 \mathrm{MV}-\mathrm{X}$ and 18 MV-X energy.Statistical analysis results of the comparison of PB and CS algorithm is demonstrated in Table 3. As it can be seen from the results related to $6 \mathrm{MV}$-X energy in Table 3, only V25 and V30 showed statistically significantly higher values in PB algorithm compared to CS algorithm $(\mathrm{p}=0.012$ and $\mathrm{p}=0.028$, respectively). As for $18 \mathrm{MV}-\mathrm{X}$ energy, again only V25 and V30 showed statistically significantly higher values in PB algorithm compared to CS algorithm $(p=0.021$ and $p=0.007$, respectively).

\section{Discussion}

Since it affects the long term survival, the importance of reducing the late cardiac side effects of RT is clear. In RT treatment planning, it becomes more important to calculate cardiac dose-volume parameters accurately for the prediction of cardiac toxicity. RT parameters that define the risk of a cardiac event are dose and irradiated volume $(1,2)$ In order to minimize cardiac mortality, Dabaja et al. used V5 and V30 parameters in their RT technique developed to reduce heart and breast doses in mediastinal RT of HL patients (3). During implementation of RT in patients with mediastinal HL, Ghalibafian et al. took into account V30 as well as MHD in intensity modulated RT (IMRT) applied for the protection of heart and coronary arteries (4). Similarly, Girinsky et al. used V30 and MHD parameters in a study comparing IMRT, 3-D conformal RT and conventional RT in HL patients with mediastinal involvement (5). Ung et al. used V25 parameter for the heart volume remaining within the RT field during chest wall irradiation in breast cancer patients who had mastectomy (6). There is not any study performed in HL patients treated with mediastinal RT which compares PB and CS algorithms in terms of cardiac dose-volume parameters. In our study, PB and CS algorithms were compared using heart V5, V25, V30 and MHD parameters in this group of patients.

Scholz et al. showed that the mean PTV dose was calculated as $8 \%$ and $1.5 \%$ higher with $\mathrm{PB}$ algorithm in the lung and head-neck tissue in which inhomogeneity is evident (7).In the study of Vanderstraeten et.al., the mean relative difference between the PB and CS algorithms using $6 \mathrm{MV}-\mathrm{X}$ energy was found as $-2.4 \%(p=0.030)$ for lung V30 parameter, $-1.0 \%(\mathrm{p}=0.008)$ for heart $\mathrm{D} 33$ parameter and $-2.04 \%(\mathrm{p}=0.105)$ for heart Dmax parameter, values being higher with $\mathrm{PB}$ algorithm compared to CS algorithm (8). In the same study, the mean relative difference between the $\mathrm{PB}$ and $\mathrm{CS}$ algorithms using $18 \mathrm{MV}$-X photon energy was found as $-0.06 \%(\mathrm{p}=0.999)$ for lung V30 parameter, $0.77 \%(\mathrm{p}=0.006)$ for heart D33 parameter, and $1.17 \%(\mathrm{p}=0.348)$ for heart Dmax parameter values being higher with PB algorithm compared to CS algorithm (8). 
Table 1. Minimum, maximum and mean \pm standard error values of V5, V25, V30 and HD parameters in the formed plans by using PB and CS algorithms for $6 \mathrm{MV}-\mathrm{X}$ photon energy

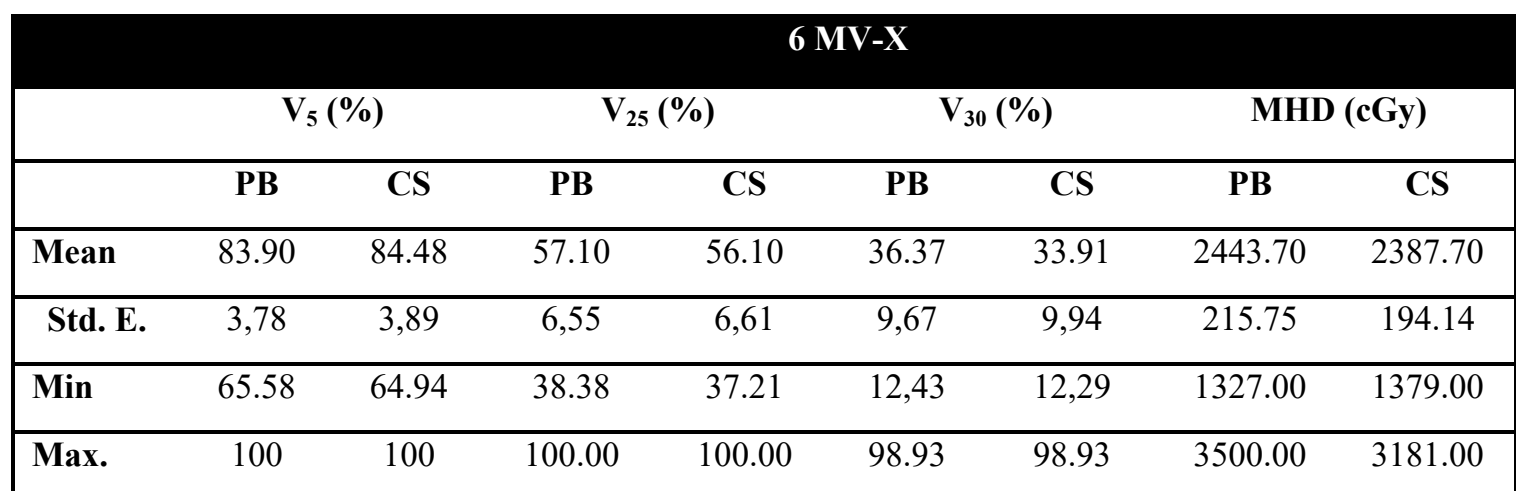

Mean, Std. E: standard error, Min: minimum, Max: maximum, V5: percent (\%) value of heart volume receiving at least 5 Gy radiation dose, V25: percent (\%) value of heart volume receiving at least 25 Gy radiation dose, V30: percent (\%) value of heart volume receiving at least 30 Gy radiation dose, MHD: Median heart dose, PB: pencil beam, CS: convolution/superposition

Table 2. Minimum, maximum and mean \pm standard error values of V5, V25, V30 and MHD parameters in the formed plans by using PB and CS algorithms for $18 \mathrm{MV}-\mathrm{X}$ photon energy

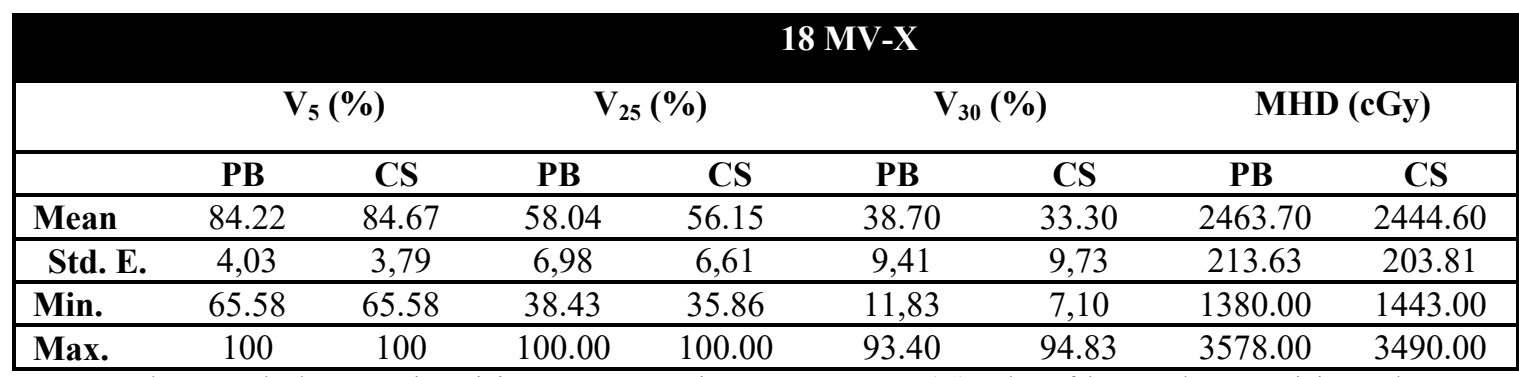

Mean, Std. E: standard error, Min: minimum, Max: maximum, V5: percent (\%) value of heart volume receiving at least 5 Gy radiation dose, V25: percent (\%) value of heart volume receiving at least 25 Gy radiation dose, V30: percent (\%) value of heart volume receiving at least 30 Gy radiation dose, MHD: Median heart dose, PB: pencil beam, convolution/superposition

Table 3. Comparison results of PB and CS algorithms in terms of cardiac dose-volume parameters by using Wilcoxon signed rank test $(\mathrm{p}<0.05$ shows statistically significant differences).

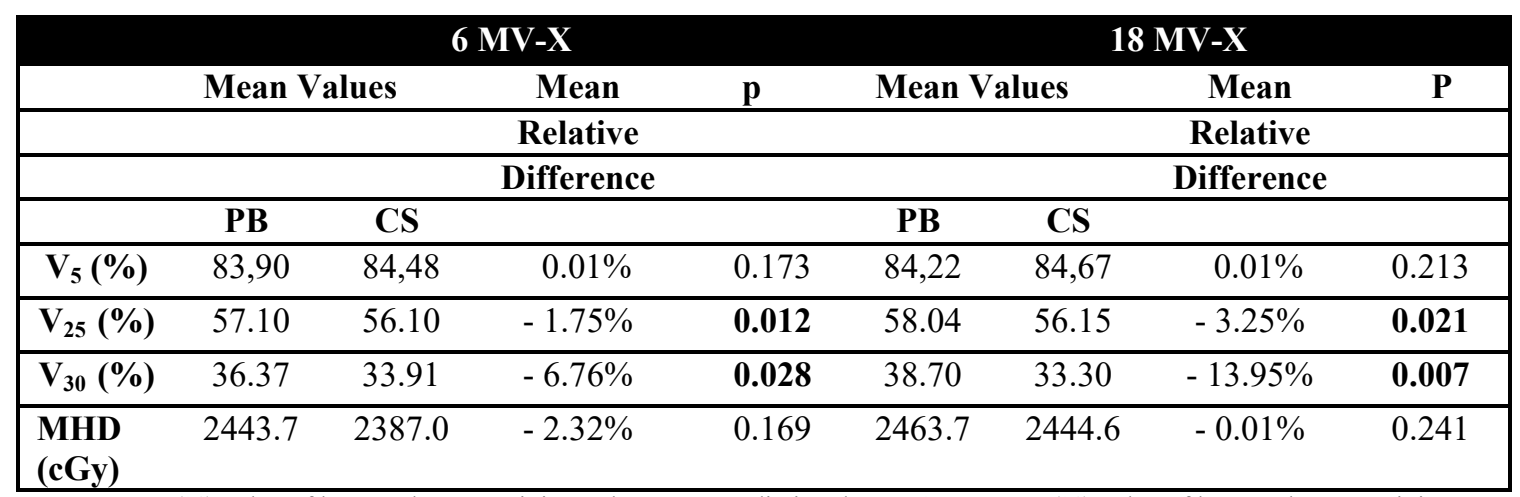

V5: percent (\%) value of heart volume receiving at least 5 Gy radiation dose, V25: percent (\%) value of heart volume receiving at least 25 Gy radiation dose, V30: percent (\%) value of heart volume receiving at least 30 Gy radiation dose, MHD: Median heart dose, PB: pencil beam, CS: convolution/superposition, Mean Relative Difference: CS value - PB value / PB value x 100 
Also in our study, significant differences were observed in favor of PB for heart V25 and V30 for 6 MV-X and $18 \mathrm{MV}-\mathrm{X}$ energy. Although the cardiac parameters used in our study is different than in Vanderstraeten et.al.'s study, they are in accordance with the results of that study.

The mean relative difference between $\mathrm{PB}$ and CS algorithms in terms of heart V25 and V30 was higher for $18 \mathrm{MV}-\mathrm{X}$ than the difference for $6 \mathrm{MV}-\mathrm{X}$ energy. This can be explained as follows: Since CS algorithm takes into account the effect of secondary electron scattering on dose distribution depending on the tissue density, it is able to calculate the dose distribution on the mediastinum-lung tissue interface more accurately compared to PB algorithm. As energy level increases, the effect of electron scattering becomes more evident

Since CS algorithm is close to Monte Carlo algorithm with regard to sensitivity in calculation and requires shoter calculation time than Monte Carlo algorithm, it can be said that CS algorithm has the balance of speed and accuracy.

\section{Conclusion}

In our study, cardiac dose-volume parameters (V25 and V30) were found to have significantly higher values in favor of PB algorithm. It was observed that these differences were more apparent for $18 \mathrm{MV}-\mathrm{X}$ than for $6 \mathrm{MV}-\mathrm{X}$ energy. These results are similar to the results obtained by Vanderstraeten et. al. who used different cardiac dose-volume parameters from our study.

The sensitivity of CS algorithm is close to Monte Carlo algorithm and calculation time is shorter. Moreover, in our study, the difference detected between $\mathrm{PB}$ and $\mathrm{CS}$ algorithms is statistically significant. Therefore, it is more suitable to use CS algorithm instead of PB algorithm in order to increase the accuracy of calculated dose distribution in HL patients who receive mediastinal RT, similarly to head-neck and lung cancer patients who have particularly evident tissue inhomogeneity within their RT fields.

Conflict of Interest: The authors declare no potential conflicts of interest with respect to the research, authorship, and/or publication of this article.

Ethical issues: All Authors declare that Originality of research/article etc... and ethical approval of research, and responsibilities of research against local ethics commission are under the Authors responsibilities. The study was completed due to defined rules by the Local Ethics Commission guidelines and audits.
Aknowledgement: None

\section{Reference}

1. Gagliardi G, Constine LS, Moiseenko V, Correa C, Pierce LJ, Allen AM, et al. Radiation dose-volume effects in the heart. International Journal of Radiation Oncology BiologyPhysics. 2010;76(3 Suppl):S77-85.

2. Hancock SL, Tucker MA, Hoppe RT. Factors affecting late mortality from heart disease after treatment of Hodgkin's disease. Jama. 1993;270(16):1949-55.

3. Dabaja BS, Rebueno NC, Mazloom A, Thorne S, Perrin KJ, Tolani N, et al. Radiation for Hodgkin's lymphoma in young female patients: a new technique to avoid the breasts and decrease the dose to the heart. International Journal of Radiation Oncology BiologyPhysics 2011;79(2):503-7.

4. Ghalibafian M, Beaudre A, Girinsky T. Heart and coronary artery protection in patients with mediastinal Hodgkin lymphoma treated with intensity-modulated radiotherapy: dose constraints to virtual volumes or to organs at risk? Radiotherapy and Oncology : Journal of the European Society for Therapeutic Radiology and Oncology. 2008;87(1):82-8.

5. Girinsky T, Pichenot C, Beaudre A, Ghalibafian M, Lefkopoulos D. Is intensity-modulated radiotherapy better than conventional radiation treatment and threedimensional conformal radiotherapy for mediastinal masses in patients with Hodgkin's disease, and is there a role for beam orientation optimization and dose constraints assigned to virtual volumes? International Journal of Radiation Oncology Biology Physics. 2006;64(1):218-26

6. Ung YC, Sixel KE, Bell C. The influence of patient geometry on the selection of chest wall irradiation techniques in post-mastectomy breast cancer patients. Radiotherapy and Oncology: Journal of the European Society for Therapeutic Radiology and Oncology. 2000;57(1):69-77.

7. Scholz C, Nill S, Oelfke U. Comparison of IMRT optimization based on a pencil beam and a superposition algorithm. Medical Physics 2003;30(7):1909-13.

8. Vanderstraeten B, Reynaert N, Paelinck L, Madani I, De Wagter C, De Gersem W, et al. Accuracy of patient dose calculation for lung IMRT: A comparison of Monte Carlo, convolution/superposition, and pencil beam computations. Medical Physics 2006;33(9):3149-58.

Copyright (C) 2016 The Author(s); This is an open-access article distributed under the terms of the Creative Commons Attribution License

(http://creativecommons.org/licenses/by/4.0), which permits unrestricted use, distribution, and reproduction in any medium, provided the original work is properly cited. All Rights reserved by international journal of Medical Science and Discovery. 